\title{
Chapter 12 \\ The Reflective Debrief: Using Students' \\ Placement Experiences to Enrich \\ Understandings of Distinct Kinds of Nutrition and Dietetic Practice
}

\author{
Lauren T. Williams, Lynda Ross, Lana Mitchell, and Katherine Markwell
}

\subsection{Introduction: Why Is Reflective Debriefing a Useful Post-practicum Intervention for Health Professional Students?}

The practicum is one of the most important components in health professional education. It is also one of the most challenging. Spending time in the acute care hospital setting can be confronting, stressful and upsetting for health professional students (Andrews et al., 2006). James and Chapman (2010) investigating student nurses' clinical placement experiences identified a key theme as 'being overwhelmed and confronted'. A systematic review of qualitative research of student nurses found that 'stress and coping' underpinned the practicum experience in this profession (Thomas, Jack, \& Jinks, 2012). Students may experience emotionally confronting situations on clinical placements (e.g. patient death) and do not necessarily receive adequate debriefing for those events (Macdonald \& Tighe, 2014). Students may carry negative emotions from these experiences, with the potential to affect their future working lives; thus it is important for the university to utilise specialised learning and teaching strategies to enable students to process their experiences.

Debriefing is a learning and teaching strategy that can be used post-practicum to ameliorate the stress associated with the placement environment and to provide students with the opportunity to develop reflective skills and knowledge in self-care and to embed practicum evaluation within the curriculum (Mackenzie, 2002).

\footnotetext{
L. T. Williams $(\bowtie) \cdot$ L. Ross $\cdot$ L. Mitchell

School of Allied Health Sciences, Griffith University, Gold Coast, QLD, Australia e-mail: lauren.williams@griffith.edu.au

K. Markwell

School of Allied Health Sciences, Griffith University, Gold Coast, QLD, Australia

Department of Clinical Sciences and Nutrition, University of Chester, Cheshire, UK
} 
Debriefing is a particular type of reflection, based on specific educational learning intentions (Mackenzie, 2002) and critical analysis of practical simulations (Dreifuerst, 2012). It has been defined as 'a purposeful reflection which can be undertaken by an individual or group' (Pearson \& Smith in Mackenzie (2002, p. 83)). Quinton and Smallbone (2010) defined reflection as 'a mental process that incorporates critical thought about an experience and demonstrates learning that can be taken forward' (p. 126). Reflection has been associated with improved critical reasoning, essential for healthcare professionals (Feather \& Fry, 2008; Wetmore, Boyd, Bowen, \& Pattillo, 2010). A review by Mann, Gordon, and Macleod (2009) identified both peer support and guidance with supervision as useful to developing reflective ability. Reflective practice enhances problem-solving abilities within changing environments (McGuire, Lay, \& Peters, 2009). Instructor-led debriefing has been found to be associated with higher student satisfaction and learning outcomes in physiotherapy students (Roh, Kelly, \& Ha, 2016).

\subsection{Placement in Nutrition and Dietetic Programmes}

Like other health professional disciplines, dietetic students undertake a significant proportion of their professional placement in the acute hospital setting at the end of their degree. For instance, the aims of the practicum courses at Griffith University are for students to be able to demonstrate entry-level practice according to the national competency standards of the Dietitians Association of Australia (2015a) and for students to have a positive experience that enhances future confidence in dietetic practice (Ross, Mitchell, \& Williams, 2017). The development of reflective skills is a key area of competency required (Dietitians Association of Australia, 2015b). Reflective practice forms the keystone of the newly graduated dietitian's credentialing as an Accredited Practising Dietitian (APD).

As health professionals, student dietitians are exposed to stressful events in the hospital setting (Macdonald \& Tighe, 2014), including bioethical issues, particularly those raised by feeding interventions (Tighe \& Mainwaring, 2013). Students are supported by supervisors on placements, and the role of the supervisor includes facilitating reflection (Burton, 2000); however several factors may compromise this role. Supervisors have competing demands and may not have sufficient time to consistently assist students' reflective skill development. Other allied health students have reported that reflective learning was not always present in the supervisory relationship (Trede \& Smith, 2012) and performing the dual role of a supervisor and assessor (Burton, 2000) could discourage full disclosure and reflection by students. This leaves students in need of support from the academic team.

However, the nature of placement is that students are distant from the academic environment. Due to competition for placements, student dietitians are placed all over Australia, away from their peers and from the academic team. This limits the ability of academic staff to assist with practicum-based learning. Griffith University staff had attempted to use online sessions to guide students in reflection during their 
placement, but this proved problematic. Some students are placed in remote areas where Internet connectivity is unreliable at best. This meant that technology-based connectivity was not possible for all students. A change in academic leadership in the discipline resulted in the 2015 final-year students being required to return to Griffith University post-placement for a week-long workshop introduced for the purposes of conducting final assessment and consolidating field-based learning. While student feedback about the workshop was positive, there was no opportunity for students to reflect formally and collectively on what they had learned in the stressful environment of the hospital setting, before seeking employment in that setting. This required a specific intervention in the post-placement curriculum.

In 2016, consequently, the post-placement workshop was modified by the academic team using design-based research principles, where development and research take place through continuous cycles of design, enactment, analysis and redesign (Edelson, 2002; van den Akker, Gravemeijer, McKenney, \& Nieveen, n.d.). Through seeking and acting on student feedback, the students became codesigners of the curriculum. This project was part of a broader initiative to improve the pedagogic consistency of the practicum with the programme curriculum (Ross et al., 2017). Three activities were added to the post-placement workshop as part of a funded project: reflection and debriefing on hospital experiences, continuing professional development goal setting and a simulated job application and interview process. This chapter describes the development, implementation and evaluation of the reflection and debrief module designed to process student learning around hospitalbased placement experiences. The aim of the reflective debrief was to provide a facilitated opportunity for students to reflect formally and collectively on their experiences in the hospital setting and to explore the implications of the reflection for working in that setting in future.

\subsection{Conceptual Framework for the Post-placement Reflection and Debrief}

Development of a reflective debrief session provided an opportunity for students to process challenging hospital experiences and develop collegial debriefing skills. Collegial debriefing has been found to be the most commonly used self-care method to assist with preventing compassion fatigue and burnout by Australian dietitians employed in the acute care setting (Osland, 2015). Innovative methods of teaching reflective practice have been used for dietetic and other health professional students, including group blogging, peer-assisted learning, simulations and portfolio (Christiansen, Buus Bøje \& Frederiksen, 2015; Ladyshewsky \& Gardner, 2008; Wright \& Lundy, 2012). Lewis (2013) summarised the evidence and application of reflective practice to allied healthcare practitioners (specifically speech therapists) and summarised several methods for teaching reflection, including written reflection and reflections on a critical incident, a clinical encounter and professional development. Griffith University dietetic students are introduced to reflective skills 
throughout the theoretical component of their programme and encouraged to keep written reflections during practicum learning. However, a review of healthcare student journaling research by Dyment and O'Connell (2011) found most studies reveal student journaling to be at a low level of reflection. More experienced practitioners use reflection-in-action in most situations (Mann et al., 2009 as cited in Lewis, 2013), whereas reflection-on-action is more suitable for novice practitioners. The dietetic post-placement reflection and debrief structure employed reflection-onaction in the form of students reflecting upon a critical incident, which could include a clinical encounter.

Delany and Watkin (2009) implemented a post-practicum discussion programme to develop critical reflection skills for physiotherapy students. This was an intensive programme of 3 hours a week discussion over a 6-week practicum programme, using critical incidents to guide reflection. Facilitators participated in a brief training session that taught them to question themselves in order to engage students as well as to move through critical reasoning stages (Delany \& Golding, 2014; Delany \& Watkin, 2009). Facilitator skill development is considered important in debriefs in interprofessional training (Di Prospero \& Bhimji-Hewitt, 2011). Delaney and Watkin's (2009) debriefs incorporated critical incidents with students, drawing on the work of Benner (1984) and Dunn and Hamilton (1986) and stages of critical reasoning. Critical incidents have been identified as an important method to develop reflective ability (Lewis, 2013). A critical incident 'is a significant situation, event or opportunity that has occurred in practice, which has the potential to provide insight or stimulate professional development' (Ash et al., 1992 as cited in Dowding, Ash, \& Shakespeare-Finch, 2011, p. 298). Critical incidents can be either positive or negative (Marquis \& Gayraud, 2002). Marquis and Gayraud (2002) conducted an exploratory study into dietetic critical incidents and identified four main areas in incidents: actors involved, positive/negative incident, work skills required/workload and impact of incident.

Critical incidents have been used to assess the clinical experiences of new graduate dietitians (Dowding et al., 2011) and to evaluate the clinical teaching experiences of healthcare students (Solomon, 2011). We were thus fairly confident that final-year dietetic students nearing graduation would be able to identify critical incidents and the stages of critical reasoning used by physiotherapy students in Delaney and Watkin's (2009) study. The four main areas described by Marquis and Gayraud (2002) were used as prompts for dietetic students to assist them to identify critical incidents and salient information for reflection and synthesis. Reflective questions were included with critical incident recall. Questions adapted from the Newcastle Reflective Inventory for documenting a significant event (Findlay, Dempsey, \& Warren-Forward, 2011) were used to prompt the students. Six key elements in a simulation debrief identified by Neill and Wotton (2011) - structured or unstructured, positive supervisor demeanour, safe and trusting environment, use of probing and cueing questions, choosing the right time for the debrief and allowing sufficient time - were incorporated into the debrief protocol. The learning objectives were: 
- To consolidate critical reflective practice abilities using a structured framework

- To develop agentic learning precepts to assist in understanding the complexities of working in the acute care environment

- To discuss any difficult or traumatic events in a supportive group environment

- To develop collegial active listening and debriefing skills

\subsection{Outline of the Reflection and Debrief Model Adopted for the Intervention}

The intervention developed was a 90-minute reflective debrief session (deliberately called a reflection and debrief to emphasise the role of reflection for students), implemented upon return to university after completion of the practicum. The module comprised two sessions and was allocated a total of 90 min on Day 3 of the post-placement week. While the theoretical basis provided by Delany and Watkin (2009) underpinned the reflection and debrief, the practical issue of access to students meant that a single intensive reflection and debrief session were used (rather than weekly sessions during practicum as in their model). Given the relative isolation of students while on placement, a group-based, face-to-face, verbal reflective debrief was chosen for the opportunity to create a supportive learning environment in which the students could process clinical experiences with clinicians, teachers and peers, to integrate and synthesise personal experiences and achieve placement closure. The two-stage approach used by Dugan, Turman, and Barnes (2017) to encourage group participants to critique personal perspectives on leadership was adapted for the reflection and debrief. The two x 45-minute stages were delivered consecutively. The first phase consisted of small group sessions conducted in a round-table seating arrangement and facilitated by dietitians experienced as clinical educators and/or in student supervision and trained to perform the reflection and debrief (referred to as small group facilitators from here). Facilitators were trained to use a non-judgemental, problem-solving approach to encourage students to explore issues prior to discussing alternate actions and solutions. Facilitators were also encouraged to focus on developing trust and sharing within the small group. Small group format was used to create an atmosphere of trust, confidentiality and honesty to facilitate discussion of difficult experiences. The round-table style was used to emphasise the importance of equal participation and sharing by all students. The session commenced with an informal icebreaker to commence communication and sharing. The plan was for each group member to generate one or two critical incident reflections on their hospital experience and to verbally explore the situation with the group and for the small group facilitator to lead group discussion of actions and possible alternatives. The critical incident phases identified by Marquis and Gayraud (2002) were included as prompts, along with a description of what would constitute a critical incident. 
Utilising a clinical supervisor to facilitate was aimed at ensuring the hospital debrief was purposeful and relevant to increase student learning. Students were instructed to identify critical incidents to prompt reflection. Mann et al. (2009) identified support, respect between group members and free expression of opinion as enabling factors for developing reflective ability. To enhance these aspects, principles formulated by Delany and Watkin (2009) to develop trust and sharing were included in the debrief. The ground rules established included confidentiality and privacy, listening first rather than solving and being non-judgemental. Trust and confidence established in the small group sessions were the foundation for the next session (allocated 45 minutes), which immediately followed the small group sessions, designed to engage the entire cohort in a facilitated discussion aimed at synthesising key themes arising from the previous sessions. The small group facilitators left the room so that the students would take the lead in speaking on behalf of their group. One spokesperson from each group contributed to the identification of themes for the entire cohort in the large group session, and these were documented and synthesised on a whiteboard by the academic facilitator. The synthesis of their learning was designed to reflect level 5 'synthesis' in Bloom's taxonomy (Anderson et al., 2001). The facilitator helped the students relate the broad themes to their future professional practice, professional development and their lifelong or 'agentic' learning. Agentic was first coined in social cognitive theory (Bandura, 2001) and in relation to learning refers to reflecting, intentionality and taking responsibility for one's learning (Billett, 2008). The large group format was designed to document themes arising from each small group, so that students could place their personal experiences and those of their colleagues into a broader context, to appreciate the commonalities and differences in experiences. To enhance the confidentiality and trust, established in small group sessions, we made a conscious decision not to audio-record either part of the debrief.

Understanding processes related to implementation is important when introducing interventions. The Medical Research Council of the United Kingdom recommends a mixed methods approach for process evaluation, combining data from quantitative and qualitative sources (Moore et al., 2015). This methodology was adopted to provide rich data to evaluate the hospital reflection and debrief and to contribute to curriculum redevelopment using design-based research (Edelson, 2002; va der Akker, nd). A comprehensive programme of impact and process evaluation data collection was planned. The data were designed to be collected from students, small and large group facilitators and the project officer during the implementation phase and analysed for intervention redesign and reimplementation. Outcome evaluation (employment success) will also be conducted; however this will be reported outside the time frame of this project. 


\subsection{Process for Developing, Implementing and Evaluating a Hospital Placement Reflection and Debrief Module}

A project officer (KM) was employed using funds from the Australian government's (then) Office of Learning and Teaching. The project was managed by the programme director (LTW) with input from the academic dietitians on the placement teaching team (LM and LR). Ethical clearance to survey students and staff was obtained from the Human Research Ethics Committee of Griffith University prior to commencement (Approval number 2014/826).

The timeline for the stages of this project is shown in Table 12.1.

\subsubsection{Development and Implementation of the Debrief}

The project officer researched the literature for appropriate models of debriefing students and discussed these with the programme director and the project team (see conceptual framework). A protocol for the hospital reflection and debrief module was developed to document the intervention clearly and provide training materials for facilitators. The protocol included information about reflective practice, the theoretical framework for the reflection and debrief, key learning objectives and a briefing paper to guide facilitation of the reflection and debrief. The project officer provided the initial group of small group facilitators with the debrief protocol and trained them in how to implement the small group debrief script. Training was delivered in 15-minute sessions either individually or in small groups.

The first iteration of the reflection and debrief module was implemented according to the protocol in June 2016, at the end of Semester 1. The reflective debrief was attended by 31 students. In the first session, students were divided into 5 groups of 5-7 students, and each group was facilitated by a dietitian (two clinical educators and three practising dietitians). Two of the small group facilitators were also

Table 12.1 Timeline for reflection and debrief module development, implementation and evaluation

\begin{tabular}{l|l}
\hline Date & Action \\
\hline Jan-Apr 2016 & $\begin{array}{l}\text { Development of the hospital placement reflection and debrief protocol and } \\
\text { script }\end{array}$ \\
\hline $\begin{array}{l}\text { Mar-May } \\
2016\end{array}$ & Plan of the process evaluation \\
\hline June 2016 & Training of debrief facilitators in the script \\
\hline June 2016 & First implementation and mixed methods evaluation \\
\hline July-Oct 2016 & Examination of the results and redesign of the reflection and debrief \\
\hline Dec 2016 & Second implementation and survey evaluation, redesign of module \\
\hline June 2017 & Third implementation and brief evaluation \\
\hline July 2017 & Reporting on results \\
\hline
\end{tabular}


employed by the university as clinical educators for hospital placements, and efforts were made not to allocate any of the students they had supervised to their groups to optimise student comfort with disclosure and protect confidentiality. Those facilitators checked the planned small group participant allocation, and any student with whom they had previously had direct involvement was reallocated to another facilitator. Group discussions were held in private spaces. Group members took turns in sharing, and exploration was encouraged by facilitators prior to the group discussing alternate actions and solutions. Each student was able to disclose one critical incident and explore it with their group in the time allocated. Students were provided with writing materials and encouraged to note down emerging themes to bring to the large group session. In the second stage of the session, all 31 students came together in a large group discussion facilitated by the project officer who was previously unknown to the students. The five small group facilitators did not attend this session so that the students would take responsibility for reporting on their group outcomes. Key learnings from each small group were reported in turn and common themes documented and synthesised on a whiteboard by the facilitator. The large group facilitator collected detailed process evaluation data during the implementation phase, as described in the evaluation section below.

Two cohorts of students undertake placement each year, one cohort per semester. After the first iteration of the reflection and debrief module in June 2016, evaluation data were considered, and an improved version of the module was implemented in December of 2016 for the 15 students completing placement in Semester 2. For the small group session, these 15 students were divided into 1 group of 7 and 1 group of 8 students. Small group facilitators were those who participated in the previous iteration and were provided with a refresher session to train them in the revised protocol. The large group session of the debrief was again facilitated by the project officer who was previously unknown to these students. Evaluation data were collected and analysed. Further adjustments were made to the debrief protocol based on staff and student evaluations. In June of 2017, 27 students attended the third iteration of the reflection and debrief module. In session 1 these 27 students were divided into 3 groups of 9 students each, using the same allocation principles as previously. The same facilitators received refresher training along with a university academic dietitian (not directly involved in placements) who was trained in the protocol. Small group discussions went for 60 minutes and the large group session for 45 minutes. This large group session was facilitated by the senior academic responsible for the hospital placement (LR).

\subsubsection{Method for Evaluation of the Debrief}

The evaluation framework and process, impact and outcome evaluation tools were developed at the time of planning. The debrief was evaluated by the project officer using a mixed methods approach that included quantitative and qualitative data. The quantitative data were analysed using descriptive statistics in SPSS version 22, and the qualitative data were thematically analysed. Detailed process evaluation data 
were collected for the first iteration of the reflection and debrief. Student satisfaction with programme materials and delivery was assessed at the end of the module via an online survey. The survey had three items, consisting of one 5-point Likert item relating to usefulness, another relating to engagement and one open-ended item. Transcripts of focus group discussions held with students at the end of the post-placement week to explore their experiences of the entire workshop were examined, and comments relevant to the reflection and debrief module were noted. Data were also collected from group facilitators in individual interviews and surveys. The small group facilitators completed qualitative surveys about their opinion on the process of the debrief, critical incidents recalled, student learning outcomes and usefulness of the session. An implementation record and reflection document were kept by the project officer, who observed session 1 and conducted session 2 . After programme redevelopment, process evaluation data were kept on the second iteration in terms of student satisfaction. Given the minor nature of the changes for the third iteration, only some process evaluation data were collected to reduce participant burden. Implementation feasibility was assessed at all three time-points.

Impact evaluation data from four sources were collected to measure whether the educational goal of learning integration had been attained. Students were required to submit an anonymous written reflection of their learning immediately after participation in the small group discussion component of the reflection and debrief. Four items explored the influence of the debrief on their perceptions of placement, learnings as a clinical dietitian, identification of any personal skills and attributes and perception of the hospital environment as their future workplace. Answers to the open-ended reflection questions were inductively coded into themes (Braun \& Clarke, 2006), with the research question of whether students perceived a benefit from the reflective debrief and whether it changed their professional learning plans. Student notes from the small group discussion session were collected by the project officer and a record made of the whiteboard summary of the discussion in the large group session. For themes captured during the debrief, the themes created by students (small group summaries) and synthesised by the facilitator (large group themes) were inductively coded and then deductively recoded into subthemes (Braun \& Clarke, 2006) related to dietetic professional attributes and experiences to investigate student learning. The subthemes produced were reviewed by all co-researchers to enhance rigour.

\subsection{Evaluation Results for the Hospital Reflection and Debrief}

The process and impact evaluation data are presented in the section that follows. The results are presented for each iteration of the hospital reflection and debrief module. Qualitative data and integrative comments are presented separately for each iteration. Given the mixed methods analysis used, the statistical data are enhanced and to some extent explained by the qualitative data expressed in direct quotes. 


\subsubsection{June 2016 Process Evaluation Results of the Reflection and Debrief Module}

The student satisfaction scores (see Table 12.2) were not as high as we had hoped for the first iteration of the module. The mean score for 'usefulness' was 2.5 out of 5 and 3.2 for 'interesting and engaging'. However, the item evaluating the hospital placement reflection and debrief asked only about the activity as a whole, so it was difficult to determine whether the students found the small group discussion or the large group discussion equally useful. Qualitative comments varied between individuals, with one student stating that '[the] individual group reflection sessions were good but the one with everyone was a waste of time', while another said '[the] smaller group sessions are probably unnecessary. We could have just had a longer, larger group session'. Transcripts of focus group discussions held at the end of the week to evaluate the entire post-placement week, while not strictly part of this evaluation, added some relevant insights. Discussions revealed that the introduction of a written examination for summative assessment at the beginning of the week had created a negative feeling among the group, which coloured the way in which students perceived the entire week including the reflection and debrief session. This suggested we had not successfully communicated the importance of the workshop activities to the students and that we needed to make a clear demarcation between assessment activities and the reflective debrief.

The small group facilitator evaluation results showed that these facilitators perceived the sessions as useful. They felt that the students reflected well in terms of both positive and negative critical incident hospital experiences. The facilitators observed that students who described having had emotion-provoking experiences (such as a patient having a cardiac arrest in their presence) were able to release some of that emotion and be supported by their peers who described similar experiences. As one facilitator said, 'I should have brought tissues'. One facilitator noted how the reflection and debrief gave students an opportunity to learn from others' experiences:

Table 12.2 Mean (SD) scores for student satisfaction on a 5-point scale for June 2016, December 2016 and June 2017 versions of the post-placement debrief

\begin{tabular}{l|l|l|l|l|l|l}
\hline & \multicolumn{3}{|l|}{$\begin{array}{l}\text { Mean (SD) usefulness of session } \\
\text { (no. of survey respondents total) }\end{array}$} & \multicolumn{3}{l}{$\begin{array}{l}\text { Mean (SD) interesting and engaging } \\
\text { (no. of survey respondents/total) }\end{array}$} \\
\cline { 2 - 8 } $\begin{array}{l}\text { Process evaluation } \\
\text { criterion }\end{array}$ & $\begin{array}{l}\text { June 16 } \\
(26 / 31)\end{array}$ & $\begin{array}{l}\text { Dec 16 } \\
(14 / 15)\end{array}$ & $\begin{array}{l}\text { June 17 } \\
(22 / 23)\end{array}$ & $\begin{array}{l}\text { June 16 } \\
(26 / 31)\end{array}$ & $\begin{array}{l}\text { Dec 16 } \\
(14 / 15)\end{array}$ & $\begin{array}{l}\text { June 17 } \\
(22 / 23)\end{array}$ \\
\hline $\begin{array}{l}\text { Debrief about } \\
\text { hospital placement } \\
\text { (both activities) }\end{array}$ & $2.5(1.27)$ & NA & $\begin{array}{l}3.77 \\
(0.9)\end{array}$ & $3.19(1.39)$ & NA & NA \\
\hline $\begin{array}{l}\text { Small group } \\
\text { debrief about } \\
\text { hospital placement }\end{array}$ & NA & $\begin{array}{l}3.18 \\
(0.6)\end{array}$ & NA & NA & $\begin{array}{l}4.36 \\
(0.5)\end{array}$ & NA \\
\hline $\begin{array}{l}\text { Whole group } \\
\text { debrief about } \\
\text { hospital placement }\end{array}$ & NA & $\begin{array}{l}2.82 \\
(0.6)\end{array}$ & NA & NA & 3.73 & NA \\
\hline
\end{tabular}

NA $=$ not asked 
... it was very useful in terms of those major things that came out to look after yourself in terms of emotional attachments to patients - having care withdrawn from patients at times when you're looking after them and having to deal with that. A lot of students hadn't come across that before, [the debrief allowed them] to see and hear someone else's experiences.

The small group facilitators felt that the reflective debrief was particularly useful to reveal commonality of the student experience and broaden individual perspectives. Ideas of how to handle patients or experiences that they may not themselves have encountered on placement were perceived as useful. Facilitators felt that students found the application of their university learning in the practice setting to be the main challenge. The small group facilitators described other students as having 'lightbulb moments' during the session where they expressed that they realised that they might have done things differently or might react differently in a similar situation in the future. Despite allocating students to facilitators from whom they had not experienced placement supervision, one questioned whether the students: '...truly opened up'. However, the project officer observed that the clinical teaching knowledge and experience of the clinical educators to be invaluable in guiding students to deeper reflections. The need to reflect on positive as well as negative experiences emerged as a subtheme under the learning process, where this facilitator described it as:

... a lovely safe environment where students opened up and could voice their reflection and experience positive or otherwise.

The large group facilitator found that it was difficult to draw responses out of the group initially, and some did not participate, but that the summary produced by those who did participate appeared effective. Themes collected on the whiteboard synthesised the four themes arising from the small group discussions. Emerging from the data was the significant role played by dietitian supervisors on placement in shaping the student experience. Several negative impacts were described by students, but there was also recognition of the importance of supervision to developing as a practitioner. By synthesising the small group learning and linking it to dietetic practice, the large group facilitator emphasised what students had achieved and learned in the small group discussions. This synthesis served both to reinforce learning and to expose small groups to the broader range of themes in order to be able to place their personal experience in context.

In terms of implementation feasibility, the teaching team met regularly to revise the post-placement week programme and material. It was imperative to have a project officer who was employed in addition to the usual academic team to drive the initial changes. While the academic team were interested in the activities, they did not feel they had the capacity to introduce the new initiatives themselves and took little ownership of the activity in the early stages. It was also apparent that the available meeting times were of insufficient length for the detailed consideration of all the results. Several decisions about the development of the module were therefore made by the project officer and the programme director, based on the process evaluation data presented and the impact evaluation data described in the next section. 


\subsubsection{June 2016 Impact Evaluation: What Did the Students Learn from the Reflection and Debrief?}

The critical incidents that the students chose to discuss during the small group debrief fell into four main themes, as shown in Table 12.3: self-management, professional identity formation, the learning process and performing dietetic work in the hospital environment. Direct quotes are not available due to the deliberate lack of audio recording. This proved to be a good decision given that several, during the session, students verbally checked that it was not being recorded.

The first theme, self-management, related to the need for students to manage their emotions in the stressful environment of a hospital placement. The high-stakes nature of being assessed on placement created stress, and some students reported being able to draw on personal reserves of resilience to manage their emotions. The second theme revealed that the experiential learning environment helped students develop their professional identity in terms of being part of a team delivering patient care. They began to develop a sense that they could make a useful contribution to patient outcomes and began to assume that sense of responsibility. In the third theme, the learning process, students described the challenge of adapting to learning within a new environment. Their learning came from supervisors, personal reflection and the challenge of the hospital situation. Students described the process of supervision as making both positive and negative contributions to their learning.

Table 12.3 Key themes noted by students as arising from their small group discussion

\begin{tabular}{l|l}
\hline Theme & Subthemes \\
\hline 1. Self-management & $\begin{array}{l}\text { 1.1 Emotional toll: managing emotions, feeling overwhelmed, } \\
\text { situational stress }\end{array}$ \\
\cline { 2 - 2 } & 1.2 Iner
\end{tabular}

1.2 Inner strength: confidence, resilience, handling conflict, motivation, persistence

1.3 Self-care: seeking support, seeking balance, conscious of own health

2. Professional identity formation

3. The learning process
2.1 Feeling like part of the treating team

2.2 Taking responsibility, making a difference to patients

2.3 Shifting from student to health professional

3.1 Learning from supervisors: taking feedback/dealing with negative feedback, acting on feedback, need to clarify expectations

3.2 Learning about yourself: reflection, learning about yourself, need to feel reassured, defining goals

3.3 Situational learning: adaptation/being flexible in response to change, being outside comfort zone/jumping in the deep end, learning on the job, steep learning curve, differences between placement sites

\footnotetext{
4. Performing dietetic work in the hospital environment
}

4.1 Teamwork: working in a team with other professionals

4.2 Communication: need for clear communication; challenges communicating with patients and family/clarification/raise issues early/dealing with conflict, documentation 
Several students recalled inconsistencies between supervisors' feedbacks, both within and across placement sites, as a source of stress. Receiving constructive feedback was felt to be important for learning. Some critical incidents reflected upon the need to adapt quickly and expressed a sense of being confronted by challenge. The final theme related to key features of performing dietetic work in the hospital environment, including the interactions involved in working within a health professional team and the need for clear communication. Students expressed the view that dealing with conflict and communication difficulties was key aspect of the work of an APD in a hospital environment. The critical incident approach revealed the central importance of communication to dietetic practice, for instance, the need to be able to draw on detailed clinical notes when something has gone wrong in patient care. Underlying all these themes is the sense that students are transformed by the hospital placement experience, in ways that might be positive or negative. In the second iteration of the module in December 2016, some new subthemes were added including the 'medical team not taking you seriously' and 'death and dying' of the patient being added to theme four. For the third iteration, no new themes or subthemes were added, but there was one additional code under the subtheme 3.1, about the supervisory relationship, with one student feeling that their supervisor was 'micro-managing' their supervision.

\subsubsection{Themes from the Documented Reflections by Individuals}

Twenty-one of the 31 participants completed the anonymous debrief reflection questions immediately following the small group session. In response to the first question, which was about whether the reflection or debrief had changed how they viewed placement, 8/21 described the debrief as not changing their view of hospital placement: 'It hasn't, because I had an enjoyable experience'. Other students described the module as useful to 'know that others had similar experience'. For some, their perspective of placement changed, as a result of the module, to be more positive through obtaining information and ideas about how to cope better in hospitals in the future. Two students noted that negative discrepancies and differences between placements had given them a negative perspective on placement. Student learning about themselves as a clinical dietitian, varied. While five students said they found nothing new, many others had discovered aspects that were in alignment with the goals of the reflection and debrief. Students recognised shared experiences with others. In response to the question about what specific skills or attributes they were now able to identify in themselves, some students found they were good at reflective practice, and others recognised their own adaptability or an ability to see the positives. Some identified clinical skills in themselves, such as good rapport and negotiation skills. Some students recognised that they were more confident than they had realised and others that they had good clinical reasoning. Others found 
areas they needed to improve to be able to work in a hospital, such as being more assertive. The final reflection question asked about how well-suited they thought they were to hospital work. The majority responded they were quite or very wellsuited to the hospital environment. Three students were not interested in working in this environment and thought there were other areas to which they were better suited.

Nine of the 15 students in the third iteration completed the anonymous reflective survey. As for the first iteration, most students felt it was useful to learn about the experiences of others, although one student was surprised by the amount of negative comments about placement. The learnings in this group were similar to those expressed by the first cohort in that they discovered that having doubts about their confidence at the outset of placement was common. In response to Q3 as to what specific skills or attributes students were now able to identify in themselves, three students identified confidence, two said communication, and two others said resilience or adaptability. Interestingly, one student noted that they had discovered they did not need to discuss their experiences. Following the debrief, all but one student considered that they were suited to working in a hospital environment. Students in the third iteration of the module reported having developed resilience and felt that the placement had made them more aware and better prepared for the possible challenges ahead in working as a clinical dietitian. Students felt their experiences had made them stronger and more empowered to move forward and were feeling very positive about working in the role of clinical dietitian. None felt they were unsuited to working in the hospital environment.

\subsubsection{Changes Made to the Post-practicum Intervention as a Result of June 2016 Evaluation}

According to design-based research principles (Edelson, 2002), we considered the June 2016 group facilitator and student feedback and reported impact on student learning in the redesign of the post-placement week before implementing the module again in December of 2016. Several key changes were made as a result. One of the most important changes was to improve our communication with the student body regarding the purpose of the activities in post-placement weeks. Communication of these messages began prior to the students commencing placement and continued throughout the 20-week placement programme in the lead up to post-placement week. We made a dedicated effort to strongly promote the importance of the programme to students so they understood that the reflection and debrief module were for their personal and career benefit and had nothing to do with any assessment processes. To further separate the post-practicum learning experiences from assessment, the examinations were redesigned from a pencil and paper format to a practice-based case study presentation. This was designed to reduce student stress around the examination and, hopefully, ameliorate the associated negativity. 
For the small group sessions, only experienced clinical educators and supervisors were engaged as facilitators due to their ability to draw detailed examples from the groups. To ensure that sufficient time was provided to discuss each issue raised by students, small group discussions were increased in duration from 45 to $60 \mathrm{~min}$, and only one critical incident was required of each participant. To encourage free sharing, students who were on placement in pairs were placed into separate groups for the small group discussions, where possible, and the importance of confidentiality was further emphasised. The large group discussion session time was decreased from 45 to $30 \mathrm{~min}$ and held in a smaller more intimate tutorial room, rather than a lecture theatre, to make discussions less formal. To support students to process any emotional reactions to the reflection and debrief, a university counsellor drop-in session was organised for immediately after the large group discussion. A final change was the creation of evaluation items to assess individually the small-group and large-group components of the hospital experience reflection and debrief on the survey.

\subsubsection{December 2016 Process Evaluation of the Module and Subsequent Changes}

Improvements to the session content and communication around the purpose of these activities resulted in much higher mean satisfaction scores, increasing from 2.5 to 3 for 'usefulness' and from 3.2 to 4.0 for 'interesting and engaging' (Table 12.2). The change to the evaluation items to reflect each part of the debrief activity showed that the majority of students found the small group section of the activity more interesting and more useful than the large group session. The qualitative comments reflected this positivity. One participant said 'I enjoyed these sessions and found them very helpful', while another remarked that the 'small group debrief was a good number of students to reflect with'. The December 2016 cohort was much smaller, and there were only two facilitators, both of whom were clinical educators. While there was only time to share one critical incident, one facilitator noted that students often wanted to share both a positive and a negative incident. The facilitators again observed a sense of commonality among students, but this cohort tended to view things in a more positive way. As one facilitator reported:

The second time that I facilitated, the group spent a lot more time discussing all the difficult
or negative aspects of placement, however I didn't feel the need to step in and re-direct or
facilitate them to reflect on more positive experiences. I felt more confidence in using
empathetic phrases and general questions ... which then ... resulted in the group being able
to put their experiences into perspective, comfort each other and in each instance [they]
were always able to get around to reflecting on the positives or 'silver linings' on their own.

The large group facilitator considered the cohort to be more comfortable and less stressed and noted that they appeared more interested in participating than the previous cohort. This may reflect the assessment changes made in response to Semester 
1, 2016 feedback or the fact that this was a smaller group in a smaller room, possibly more open to sharing emotionally than the previous cohort group. After this second implementation, we made some minor refinements prior to implementation on June 1, 2017. The total time for the session was extended from 90 to 105 min to allow sufficient time for discussion. For the small group session, the briefing paper for the group facilitators was streamlined to improve flow and to provide options/ examples to help probe/prompt students if needed. This version of the module was still organised and facilitated by the project officer. However, staff were becoming more engaged through facilitating the small group discussion. However, the activity was still perceived as belonging to a special project rather than the programme.

\subsubsection{June 2017 Process Evaluation of the Module and Final Changes}

Organisationally, the project officer completed her role, and in the future the senior academic dietitian responsible for coordinating hospital placement will be responsible for the reflection and debrief. Students in this post-placement cohort were satisfied with the session, reflected in the evaluation scores. The 'usefulness' rating increased from a mean score of 3.0 to 3.8, approaching 'very useful' (4.0). Only two students reported the activity as 'not useful', showing a more consistent positive score than for previous implementation rounds. Qualitative comments were all positive in tone, as highlighted by the following examples:

A great idea to have included which helped me to identify that my situation was not whole (sic) reflective of myself, but that of the hospital ...

It was a safe space to share information with peers

It was a very helpful way to normalize some of the experiences that I had on placement by hearing everyone else's experiences.

The small group facilitators found the process useful and felt it worked well with an appropriate number of participants in each group. They found the students to be emotionally strong and very insightful regarding their experiences on placement. They felt the students approached the sessions with an unexpected level of maturity and that, despite raising of negative experiences initially, as a group, they were very reflective and supportive of each other and able to highlight the positive outcomes and opportunities for learning and growth. The small group facilitators found that the main critical incidents raised related to students not feeling respected by busy supervisors, feeling that supervisors were over critical and being supervised by multiple supervisors. These facilitators reported that the students had good insight into the need to manage the expectations of their supervisors, as well as coping with their own drop-in confidence and the need to rebuild it. 
The large group facilitator found that students believed that sharing experiences had helped to put their own experiences into perspective and hearing the experiences of others had made them feel 'normal' and no longer blame themselves for any negative experiences. Students drew on the positives, and, although they obviously found the hospital placement challenging, their experiences helped them to learn and to grow. The debrief activity was by now seen as a feasible and important part of the post-placement curriculum. After this iteration, the only changes made to the protocol were that time for students to complete the written reflection was added into the time allocation for the small group session.

\subsection{Discussion}

Evaluation of the reflection and debrief module developed for this project demonstrated how students can be assisted to process learning experiences undertaken in the challenging environment of acute hospital care. The dietetic students participating in this reflection and debrief were interested in reflecting on their own experiences and in hearing about the practicum experience of their peers, providing that they could learn something from the experience themselves. This qualified acceptance of the activity is reflected in the findings of a study of health professional student preferences for post-practicum intervention strategies (Cain et al. 2018). The findings of that survey showed that the proportion of nursing and midwifery students $(33 \%)$ who reported to be very interested in the 'opportunity to share and discuss with peers' was higher than for allied health students (including dietitians) $(25 \%)$ or students of medicine $(9.5 \%)$ (Cain et al., 2018).

Kolb and Kolb (2005) have summarised the need for reflection in higher education. Reflection is needed for many learning styles and assists in consolidation and integration of experiential learning experiences (Kolb \& Kolb, 2005). Healthcare students undertake experiential learning in long periods of work-based learning. The reflective debrief was undertaken with the aim to enhance student learning in this setting. Using a university-based reflection gives all students an equitable opportunity to ensure that they undertake reflection. Trede and Smith (2012) have found that the quality of within-placement opportunities for verbal guided reflection may depend on the student's placement supervisor. Given that negative student experiences may be related directly to a supervisory relationship of placement (Lew, Cara, \& Richardson, 2007), university-based reflection allows them to be discussed and processed. Other healthcare disciplines have similarly noted the benefit of a guided, reflective, group debrief, including physiotherapy (Delany \& Watkin, 2009), nursing (Platzer, Blake, \& Ashford, 2002) and occupational therapy students in Australia (Mackenzie, 2002) and the United Kingdom (Nicholls \& Mackenzie, 2006).

The reflection and debrief module allowed students to reflect on critical incidents that occurred during their hospital placements and, with the assistance of skilled facilitators, to process those incidents according to specified learning objectives. Facilitators were clinical educators specifically hired to support the acute care train- 
ing of healthcare students (Fairbrother, Madelyn, Blackford, Nagarajan, \& McAllister, 2016; Ferguson, Haantjens, \& Milosavljevic, 2014) and are in regular contact with students on placement and oversee their field-based learning and teaching. Their role gives them knowledge of the hospital setting and a unique perspective of the environment, challenges and perspectives of both clinical supervisors and students which helps them to interpret the students' critical incidents. This background gives the reflective debrief the advantage of having experts guiding and reframing supervisory issues for students to inform and enhance students' future careers. This could assist with retaining graduate healthcare practitioners within the hospital setting and assist them in their own future supervisory relationships and practice. An advantage of conducting the reflection and debrief in the group setting is that students can learn from other students' experiences and reflections, which could be considered a form of peer-assisted learning (Ladyshewsky \& Gardner, 2008).

Several students participating in the debrief described incidents arising in the hospital setting that took an emotional toll on them. The nursing profession has used reflective debriefs to enhance new graduate nurses' psychosocial support (Mangone, King, Croft, \& Church, 2014) and assist paediatric nurses (Maloney, 2012) to process traumatic experiences and reduce compassion fatigue. When undertaking the dietetic student reflection of acute care experiences, it was important to be cognisant of student emotional well-being as well as reflective learning. As a result of the challenging experiences described by students during the first debrief at our university, the programme director organised a university counsellor to be available for student consultations directly after subsequent debriefs. In this way, the reflective debrief has the potential to assist students do more than enhance their learning; they can also be assisted with resolving emotional experiences through access to support services. One small group reflection for medical residents described in the literature was considered a wellness initiative (Wen et al., 2013). The potential to enhance emotional wellness requires future investigation with respect to dietetic and other healthcare students.

The design-based research approach applied by the academic team to the programme curriculum proved useful. Despite the extensive research undertaken by the project officer and the careful theoretical framework applied to planning the reflection and debrief, the first iteration did not score as highly as we had hoped in terms of student satisfaction. Careful and detailed evaluation revealed possible explanations for this and guided the redevelopment for the second iteration of the module. The student-informed redevelopment of the module led to higher student satisfaction and more positive comments the second time the module was implemented. Qualitative comments in the third implementation were more positive and student satisfaction even higher again, despite fewer programmatic changes, suggesting that something else may have influenced these results. The first two versions of the module, conducted in 2016, were delivered to students who commenced their four-year degree in 2013. Students undertaking the third version of the module in 2017 commenced their degree in 2014, which saw several changes to the entire programme 
curriculum - so that these students experienced a significantly different degree structure and content. The maturity and resilience of the third iteration students observed by the small group facilitators may have been a result of these curriculum changes. Or perhaps this reflects an exceptional group of students. Another potential explanation is that this large group session was conducted by the clinical dietetic lecturer, which may have changed the process. Perhaps this facilitator was more able to draw out positive emotional themes, or, alternatively, students were less willing to share negative experiences with her. Module evaluation for subsequent cohorts will indicate which explanation is most likely.

Several key lessons were learned by the nutrition and dietetic team through the process of conducting this project. The first lesson was that developing even a short intervention in an evidence-based way requires significant dedicated resources. Given the team was short-staffed at the time, the project would not have been conducted, or conducted to the same level of quality, had we not had the funds to employ a project officer. We were very fortunate in our choice of project officer, given her high-level abilities, persuasive manner and the fact that she spent a lot of time in consultation with key academics. A second lesson was to avoid introducing or changing assessment at the same time as introducing another new initiative. The introduction of a final exam into the post-placement week generated resentment within the first cohort of students, masking the potential for the reflection and debriefing module to be successful. Students saw that module as another form of assessment, rather than recognising the benefit to them. This highlighted the importance of clearly communicating to students, who are in the process of transitioning to being professionals, the difference between doing something because it is assessable or compulsory and doing something that will enhance learning for their future career. Finally, the evaluation data highlighted the importance of obtaining both quantitative and qualitative feedback on new activities being introduced and the need to measure each component of a new activity separately. Qualitative interviews with key stakeholders in implementing the module proved vital to interpreting the quantitative data. It was also important not to abandon the module after the first attempt but to persevere with improving it, based on feedback.

The critical incidents discussed highlight other actions that need to be undertaken. Future training programmes will feedback these results to fieldwork supervisors. It may surprise some supervisors to hear the negative ways in which students perceive what may be intended as constructive feedback. We can also work to assist students in managing their emotions and improving their resilience prior to undertaking placement and in fact have already undertaken steps to do this by introducing a pre-placement session with a university counsellor on building resilience. Facilitating this learning requires great skill and requires the facilitators to be experienced in the hospital placement. We will also use the qualitative themes to brief the students better about the types of learning experiences they might expect prior to placement. 


\subsection{Conclusion}

The current workforce profile in Australian dietetics sees hundreds of new graduates competing for each advertised position in the hospital sector, which is still the major employer (Health Workforce Australia, 2014). The ability of final-year dietetic students to reflect on their practice has been found to be important in developing the critical thinking and clinical reasoning that is essential for success in the hospital setting (Palermo, Walker, Brown, \& Zogi, 2009). Skills in reflective learning, therefore, have the potential to provide graduates with an 'edge' in obtaining these highly contested positions and making a successful transition to the hospital workforce. The theoretical framework and design-based research approach taken by the nutrition and dietetic team at Griffith University achieved the development of a module aimed at encouraging final-year student dietitians to reflect critically on incidents occurring in their placement experience, in a safe, expert-guided environment. The critical incidents described provide insights on the nature of how the hospital placement is experienced by students in a transformational environment and were used to guide curriculum redevelopment. Successive iterations of the module showed stepped improvements in student satisfaction. The third version of the reflection and debrief is now seen as a key component of the post-placement week and is embedded in the programme curriculum. Now that the programme is stable, the next step is to proceed to outcome evaluation and measure how the activity influences graduate career employability.

Acknowledgements Thank you to Marie-Claire O'Shea who did some of the original work on this project. We also thank the students and group facilitators for the time taken to contribute to the evaluation surveys.

\section{References}

Anderson, L. W., Krathwohl, D. R., Airasian, P. W., Cruikshank, K. A., Mayer, R. E., Pintrich, P. R., et al. (2001). A taxonomy for learning, teaching, and assessing: A revision of Bloom's taxonomy of educational objectives. New York: Pearson, Allyn \& Bacon.

Andrews, G., Brodie, D. A., Andrews, J. P., Hillan, E., Thomas, B. G., Wong, J., et al. (2006). Professional roles and communications in clinical placements: A qualitative study of nursing students' perceptions and some models for practice. International Journal of Nursing Studies, $43,861-874$.

Bandura, A. (2001). Social cognitive theory: An agentic perspective. Annual Review of Psychology, $52,1-26$.

Benner, P. (1984). From novice to expert: Excellence and power in clinical nursing practice. Menlo Park, CA: Addison Wesley.

Billett, S. (2008). Learning throughout working life: A relational interdependence between social and individual agency. British Journal of Education Studies, 55(1), 39-58.

Braun, V., \& Clarke, V. (2006). Using thematic analysis in psychology. Qualitative Research in Psychology, 3(2), 77-101.

Burton, S. (2000). A critical essay on professional development in dietetics through a process of reflection and clinical supervision. Journal of Human Nutrition and Dietetics, 13(5), 323-332. 
Cain, M., Le, A. H., \& Billett, S. (2018). Ch 2: Sharing stories and building resilience: Student preferences and processes of post-practicum interventions. In Augmenting health and social care students' clinical learning experiences: Outcomes and processes. New York: Springer.

Christiansen, S., Buus Bøje, R., \& Frederiksen, K. (2015). The use of problem-and simulationbased learning: The student's perspective. Nordic Journal of Nursing Research, 35(3), 186-192.

Delany, C., \& Golding, C. (2014). Teaching clinical reasoning by making thinking visible: An action research project with allied health clinical educators. BMC Medical Education, 14(1), 1.

Delany, C., \& Watkin, D. (2009). A study of critical reflection in health professional education: 'Learning where others are coming from'. Advances in Health Sciences Education, 14(3), 411-429. https://doi.org/10.1007/s10459-008-9128-0

Di Prospero, L., \& Bhimji-Hewitt, S. (2011). Learning is in the facilitation: Faculty perspectives with facilitated teaching and learning-Recommendations from informal discussions. Journal of Allied Health, 40(4), 61E-65E.

Dietitians Association of Australia. (2015a). National competency standards for dietitians in Australia. URL: https://daa.asn.au/wp-content/uploads/2017/01/NCS-Dietitians-Australiawith-guide-1.0.pdf (20 October 2017).

Dietitians Association of Australia. (2015b). Preparing for the DAA professional examinations in dietetics: Guide to mentoring and continuing professional development for dietetic skills recognition candidates (pp. 1-31). URL: https://daa.asn.au/wp-content/uploads/2016/06/Guideto-Mentoring-CPD-2016.pdf (July 2017).

Dowding, K., Ash, S., \& Shakespeare-Finch. (2011). Using critical incident interviews to identify the mental health knowledge, skills and attitudes of entry-level dietitians. Nutrition \& Dietetics, 68, 297-304.

Dreifuerst, K. T. (2012). Using debriefing for meaningful learning to foster development of clinical reasoning in simulation. Journal of Nursing Education, 51(6), 326-333.

Dugan, J. P., Turman, N. T., \& Barnes, A. C. (2017). Ch 1: Our evolving understanding of leadership. In Leadership theory: A facilitators guide for cultivating critical perspectives. San Francisco: Jossey-Bass.

Dunn, W. R., \& Hamilton, D. D. (1986). The critical incident technique-a brief guide. Medical Teacher, 8(3), 207-215.

Dyment, J. E., \& O'Connell, T. S. (2011). Assessing the quality of reflection in student journals: A review of the research. Teaching in Higher Education, 16(1), 81-97.

Edelson, D. C. (2002). Design research: What we learn when we engage in design. Journal of the Learning Sciences, 11, 105-121.

Fairbrother, M., Madelyn, N., Blackford, J., Nagarajan, S. V., \& McAllister, L. (2016). A new model of clinical education to increase student placement availability: The capacity development facilitator model. Asia-Pacific Journal of Cooperative Education, 17(1), 45-59.

Feather, A., \& Fry, H. (2008). Chapter 23: Key aspects in teaching and learning in medicine and dentistry. In H. Fry, S. Ketteridge, \& S. Marshall (Eds.), A handbook for teaching and learning in higher education: Enhancing academic practice (3e). London: Kogan Page.

Ferguson, A., Haantjens, A., \& Milosavljevic, M. (2014). Evolution of the clinical educator role to increase student placement capacity: From traditional to innovative. Nutrition \& Dietetics, $71,51-56$.

Findlay, N., Dempsey, S., \& Warren-Forward, H. (2011). Development and validation of reflective inventories: Assisting radiation therapists with reflective practice. Journal of Radiotherapy in Practice, 10(1), 3-12.

Health Workforce Australia. (2014). Australia's health workforce series - Dietitians in focus. Canadian Journal of Dietetic Practice and Research, 75(4), 173-179.

James, A., \& Chapman, Y. (2010). Preceptors and patients - The power of two: Nursing student experiences on their first acute clinical placement. Contemporary Nurse, 34(1), 34-47. https:// doi.org/10.5172/conu.2009.34.1.034

Kolb, A. Y., \& Kolb, D. A. (2005). Learning styles and learning spaces: Enhancing experiential learning in higher education. Academy of Management Learning \& Education, 4(2), 193-212. 
Ladyshewsky, R. K., \& Gardner, P. (2008). Peer assisted learning and blogging: A strategy to promote reflective practice during clinical fieldwork. Australasian Journal of Educational Technology, 24(3), 241-257.

Lew, N., Cara, E., \& Richardson, P. (2007). When fieldwork takes a detour. Journal Occupational Therapy In Health Care, 21(1-2), 105-122.

Lewis, A. V. (2013). Reflective practice: What is it and how do I do it? Journal of Clinical Practice in Speech-Language Pathology, 15(2), 70-74.

Macdonald, J., \& Tighe, B. (2014). Student dietitians' experiences of patient death whilst on placement. European Journal of Nutrition \& Food Safety, 4(3), 181-182.

Mackenzie, L. (2002). Briefing and debriefing of student fieldwork experiences: Exploring concerns and reflecting on practice. Australian Journal of Occupational Therapy, 49(2), 82-92.

Maloney, C. (2012). Critical incident stress debriefing and pediatric nurses: An approach to support the work environment and mitigate negative consequences. Pediatric Nursing, 38(2), 110-113.

Mangone, N., King, J., Croft, T., \& Church, J. (2014). Group debriefing: An approach to psychosocial support for new graduate registered nurses and trainee enrolled nurses. Contemporary Nurse, 20(2), 248-257.

Mann, K., Gordon, J., \& Macleod, A. (2009). Reflective practice in health professions education: A systematic review. Advances in Health Science Education, 14, 595-621.

Marquis, M., \& Gayraud, H. (2002). Exploring clinical dietitians' day-to-day practice through the critical incident technique. Journal of the American Dietetic Association, 102(10), 1461-1465.

McGuire, L., Lay, K., \& Peters, J. (2009). Pedagogy of reflective writing in professional education. Journal of the Scholarship of Teaching and Learning, 9(1), 93-107.

Moore, G. F., Audrey, S., Barker, M., Bond, L., Bonell, C., Hardeman, W., et al. (2015). Process evaluation of complex interventions: Medical Research Council guidance. BMJ, 350, h1258. https://doi.org/10.1136/bmj.h1258

Neill, M. A., \& Wotton, K. (2011). High-fidelity simulation debriefing in nursing education: A literature review. Clinical Simulation in Nursing, 7, e161-e168.

Nicholls, L., \& Mackenzie, A. (2006). Ch 13. Transforming experience into learning: Briefing and debriefing for practice learning. In T. Lorezono, M. Duncan, H. Buchanan, \& A. Alsop (Eds.), Practice and service learning in occupational therapy: Enhancing potential in context (pp. 187-199). Brisbane, Australia: John Wiley \& Sons.

Osland, E. J. (2015). An investigation into the professional quality of life of dietitians working in acute care caseloads: Are we doing enough to look after our own. Journal of Human Nutrition and Dietetics, 28(5), 493-501.

Palermo, C., Walker, K. Z., Brown, T., \& Zogi, M. (2009). How dietetics students like to learn: Implications for curriculum planners. Nutrition \& Dietetics, 66(2), 108-112.

Pearson and Smith cited in Mackenzie, L. (2002), Briefing and debriefing of student fieldwork experiences: Exploring concerns and reflecting on practice. Australian Occupational Therapy Journal, 49, 82-92.

Platzer, H., Blake, D., \& Ashford, D. (2002). An evaluation of process and outcomes from learning through reflective practice groups on a postregistration nursing course. Journal of Advanced Nursing. 2000, 31(3), 689-695.

Quinton, S., \& Smallbone, T. (2010). Feeding forward: Using feedback to promote student reflection and learning-a teaching model. Innovations in Education and Teaching International, 47(1), 125-135.

Roh, Y. S., Kelly, M., \& Ha, E. H. (2016). Comparison of instructor-led versus peer-led debriefing in nursing student. Nursing and Health Sciences, 18, 238-245.

Ross, L. J., Mitchell, L. J., \& Williams, L. T. (2017). Is it possible to enhance the confidence of student dietitians prior to professional placements? A Design -based research model. Journal of Human Nutrition and Dietetics. https://doi.org/10.1111/jhn.12479. [Epub ahead of print].

Solomon, P. (2011). Student perspectives on patient educators as facilitators of interprofessional education. Medical Teacher, 33(10), 851-853. https://doi.org/10.3109/0142159X.2010.530703 
Thomas, J., Jack, B. A., \& Jinks, A. M. (2012). Resilience to care: A systematic review and metasynthesis of the qualitative literature concerning the experiences of student nurses in adult hospital settings in the UK. Nurse Education Today., 32, 657-664.

Tighe, B., \& Mainwaring, J. (2013). The bioethical experiences of student dietitians on their final clinical placement. Journal of Human Nutrition and Dietetics, 26(2), 198-203.

Trede, F., \& Smith, M. (2012). Teaching reflective practice in practice settings: Students' perceptions of their clinical educators. Teaching in Higher Education, 17(5), 615-627.

van den Akker, J., Gravemeijer, K., McKenney, S., \& Nieveen, N. (n.d.) Educational Design Research. Netherlands Organization for Scientific Research, Program Council for Educational Research (NWO/PROO); 1-245. URL: http://international.slo.nl/publications/edr/

Wen, L. S., Baca, J. T., O’Malley, P., Bhatia, K., Peak, D., \& Takayesu, J. K. (2013). Implementation of small-group reflection rounds at an emergency medicine residency program. Journal of the Canadian Association of Emergency Physicians, 15(3), 175-177.

Wetmore, A. O. K., Boyd, L. D., Bowen, D. M., \& Pattillo, R. E. (2010). Reflective blogs in clinical education to promote critical thinking in dental hygiene students. Journal of Dental Education, 74(12), 1337-1350.

Wright, L., \& Lundy, M. (2012). Blogging as a tool to promote reflection among dietetic and physical therapy students during a multidisciplinary international service-learning experience. Journal of Allied Health, 41(3), 73E-78E. 